\title{
Benign Triton Tumor
}

National Cancer Institute

\section{Source}

National Cancer Institute. Benign Triton Tumor. NCI Thesaurus. Code C121687.

A very rare intraneural mass in a large nerve characterized by the interposition of mature skeletal muscle fibers and nerve fibers. 